\title{
Age-related differences in features associated with polycystic ovary syndrome in normogonadotrophic oligo-amenorrhoeic infertile women of reproductive years
}

\author{
Helen Bili ${ }^{1,2}$, Joop Laven ${ }^{1}$, Babek Imani ${ }^{1}$, Marinus J C Eijkemans ${ }^{3}$ and Bart C J M Fauser ${ }^{1}$ \\ ${ }^{1}$ Division of Reproductive Medicine, Department of Obstetrics and Gynecology, Erasmus University Medical Center Rotterdam, Rotterdam, \\ The Netherlands, ${ }^{2}$ First Department of Obstetrics and Gynecology, Hippokration General Hospital, Thessaloniki, Greece and ${ }^{3}$ Center for Clinical Decision \\ Sciences, Department of Public Health, Erasmus University Medical Center Rotterdam, Rotterdam, The Netherlands \\ (Correspondence should be addressed to J S E Laven, Division of Reproductive Medicine, Department of Obstetrics and Gynecology, Erasmus University \\ Medical Center, Dr Molewaterplein 40, 3015 GD Rotterdam, The Netherlands; Email: laven@gyna.azr.nl)
}

\begin{abstract}
Objective: To assess the effect of age on clinical, endocrine and sonographic features associated with polycystic ovary syndrome (PCOS) in normogonadotrophic anovulatory infertile women of reproductive years.

Design: Cross-sectional study.

Methods: Four hundred and seventy-two oligo-amenorrhoeic infertile patients, presenting with normal FSH and oestradiol concentrations, aged 17-42 years underwent a standardised initial evaluation including: cycle history, body mass index, waist-to-hip ratio and transvaginal ultrasound scanning of ovaries. Fasting blood samples were obtained for extensive endocrine evaluation. Cycle duration, serum levels of gonadotrophins, androgens, oestradiol, insulin, glucose, inhibin B as well as mean number of follicles, ovarian volume and ovarian stroma echogenicity were assessed.

Results: Older women had significantly lower LH and androgen and inhibin B serum levels. Similarly, older women presented with a reduced number of ovarian follicles. Age was inversely correlated with cycle duration $(r=-0.112, P=0.02)$, LH $(r=-0.154, P=0.001)$, testosterone $(r=-0.194$, $P=0.001)$, androstenedione $(r=-0.170, P=0.001)$, dehydroepiandrosterone $(r=-0.157$, $P=0.001)$, insulin $(r=-0.126, P=0.02)$, inhibin $\mathrm{B}(r=-0.118, P=0.03)$ serum levels and mean follicle number $(r=-0.100, P=0.03)$. A positive correlation was observed between age and glucose to insulin ratio $(r=0.138, P=0.009)$.

Conclusions: Advanced age in normogonadotrophic anovulatory infertile women is associated with lower LH and androgen levels and with a decreased number of ovarian follicles. Although during reproductive years observed differences are relatively small, these age-related changes may affect the observed incidence of PCOS.
\end{abstract}

European Journal of Endocrinology 145 749-755

\section{Introduction}

Demographic and clinical studies show that various cycle characteristics change with age in normoovulatory women of reproductive age $(1,2)$. Menstrual cycles become shorter, due to decreasing follicular phase length (3). After the age of 35, follicle-stimulating hormone (FSH) levels begin to increase together with a subtle decrease in inhibin $B$ without simultaneous changes in oestradiol $\left(\mathrm{E}_{2}\right)(4-6)$. Moreover, a steady gradual decline with age has been observed in serum androgens (7). Finally, the number of antral follicles assessed during the early follicular phase has recently been shown to decrease with advanced age (8).

The diagnosis of polycystic ovary syndrome (PCOS) in anovulatory infertile women is usually based on clinical characteristics in combination with hormonal and sonographic evaluation. Although hyperandrogenaemia seems to play a central role, no agreement has been reached so far concerning criteria required for diagnosis $(9,10)$ and the age of the patients is not taken into account. Preliminary observations suggest that a gradual normalisation of menstrual cycle abnormalities occurs in PCOS patients with increasing age $(11,12)$. In contrast, other investigators (13) could not confirm such changes. Moreover, some studies recorded a decline in serum androgen levels with age in hyperandrogenic PCOS patients as well as in controls $(14,15)$. Hence, ignoring the patient's age may result in over- or under-diagnosis of the syndrome. Notwithstanding the extensive study of PCOS, little information is available concerning the potential effect of age 
especially on the extent of cycle abnormality, body weight and hyperandrogenaemia. The objective of the present study was to assess the effect of increasing age on clinical, endocrine and sonographic features associated with PCOS in a large cohort of normogonadotrophic oligo-amenorrhoeic infertile patients of reproductive years.

\section{Materials and methods}

\section{Subjects}

Four hundred and seventy-two infertile women, attending our fertility clinic between February 1993 and September 1999, presenting with oligomenorrhoea (interval between vaginal bleeding $>35$ days and $\leq 6$ months) or amenorrhoea (bleeding interval $>6$ months) were enrolled in this cross-sectional study. The study protocol was approved by the human subject committee of the Erasmus University. Additional inclusion criteria were: (i) FSH levels within normal limits (1-10 IU/l), and (ii) spontaneous or progestogeninduced withdrawal bleeding $(9,16)$. Exclusion criteria were: (i) serious illnesses such as kidney, liver, heart, vascular, thyroid diseases, or diabetes mellitus (fasting glucose $>6 \mathrm{nmol} / \mathrm{l}$ ), (ii) use of exogenous hormones, (iii) hyperprolactinaemia (PRL $>15.5 \mu \mathrm{g} / \mathrm{l}$ ), (iv) periovulatory $\mathrm{E}_{2}$ levels $(>400 \mathrm{pmol} / \mathrm{l})$, or $(\mathrm{v})$ luteal phase progesterone levels $(>15 \mathrm{nmol} / \mathrm{l})$.

Standardised clinical screening and transvaginal sonography (TVS) were performed and a fasting blood sample was obtained on a random day. Clinical screening included physical examination, menstrual and reproductive history, body mass index (BMI), waistto-hip ratio (WHR), surgery and previous medication. BMI was calculated as weight $(\mathrm{kg}) /$ height squared $\left(\mathrm{m}^{2}\right)$. WHR was defined as the minimum circumference at the waist divided by the maximum extension over the buttock, and was utilised as an index for different patterns of body fat distribution. The contribution of BMI and WHR on the effect of age on the studied parameters was evaluated because overweight is more common in older women.

Endocrine screening included serum measurements of $\mathrm{FSH}$, luteinising hormone $(\mathrm{LH}), \mathrm{E}_{2}$, progesterone, 17-hydroxyprogesterone (17-OHP), testosterone, androstenedione (AD), dehydroepiandrosterone (DHEAS), sex hormone binding globulin (SHBG), free androgen index $($ FAI $=$ Testosterone $\times 100 /$ SHBG $)$, insulin, glucose, inhibin B and cortisol. Fasting blood samples were taken by venepuncture between 0800 and $1000 \mathrm{~h}$. Serum was obtained by centrifugation and frozen in aliquots at $-20{ }^{\circ} \mathrm{C}$, for subsequent analysis.

TVS screening included assessment of the ovarian stroma echogenicity (arbitrarily classified from 1 to 3 ), ovarian volume $(\mathrm{ml})$, and total number of antral follicles (both ovaries), as previously described $(9,17)$. Mean follicle number and mean ovarian volume were calculated as the addition of left and right, divided by two and total stroma count was the stroma score addition of the left and the right ovary. For reasons of comparison PCOS was defined as elevated androgens $(\mathrm{FAI}>4.5)$ and polycystic ovaries (mean ovarian volume $>10.8 \mathrm{ml}$ ) (NIH consensus meeting, NIEHS Durham, NC, USA, September 2000) (9).

\section{Hormone assays}

Serum LH and FSH levels were measured by immunoradiometric assay (Medgenix, Fleurus, Belgium), and $\mathrm{E}_{2}$, testosterone, $\mathrm{AD}$, SHBG and DHEAS were determined using RIA kits (Diagnostic Products Corp., Los Angeles, CA, USA) as described previously (18). Insulin was measured by two-site chemiluminescent enzymelabelled immunometric assay (Diagnostic Products Corp.). For inhibin B assays a dimeric immunoassay kit, solid phase sandwich ELISA (Serotec, Kidlington, Oxford, UK) was used (19). Intra- and interassay coefficients of variation were less than $5 \%$ and $15 \%$ for $\mathrm{LH}$, less than $3 \%$ and $8 \%$ for $\mathrm{FSH}$, less than $4 \%$ and $7 \%$ for $\mathrm{E}_{2}$, less than $6 \%$ and $7 \%$ for testosterone, less than $8 \%$ and $11 \%$ for $\mathrm{AD}$, less than $7 \%$ and $8 \%$ for DHEAS, less than $6 \%$ and $8 \%$ for SHBG, less than $16 \%$ and $17 \%$ for progesterone, less than $6 \%$ and $10 \%$ for insulin, less than $1 \%$ and $2 \%$ for glucose, less than $8 \%$ and $14 \%$ for inhibin B and less than $9 \%$ and $10 \%$ for cortisol respectively

\section{Statistical analysis}

All data were analysed for normality of distribution. Pearson's correlation test was used when data were distributed normally. If not, Spearman's rank correlation test was performed. Covariance analysis was used to describe the linear relationship between variables while controlling for the effects of one or more confounding variables. Student's t-test or MannWhitney $U$ test were used to compare the means between subsets of data. The two-tailed $P$ value of $<0.05$ was used to define statistical significance.

\section{Results}

A total of 472 patients ranging between 17 and 42 years of age (28.5 \pm 4.5 years) were evaluated in this study. Clinical, endocrine and ultrasound characteristics of these 472 women are presented in Table 1. Women were also grouped by age: $17-29$ and $\geq 30-42$ years. Differences for LH, LH/FSH ratio, testosterone, AD, DHEAS, mean number of follicles and the percentage of PCOS subjects were found to be statistically significant (Table 1).

In the study population, age was inversely correlated with cycle duration, LH, testosterone, AD, DHEAS, FAI, SHBG, insulin, inhibin B levels and mean follicle number (Table 2). Bivariate correlations between age 
Table 1 Clinical, endocrine and sonographic features (mean \pm S.D.) in 472 normogonadotrophic oligo-amenorrhoeic infertile patients, and separately for women below or above 30 years of age.

\begin{tabular}{|c|c|c|c|c|}
\hline & $\begin{array}{l}\text { Overall group } \\
\qquad(n=472)\end{array}$ & $\begin{array}{c}17-29 \text { years } \\
(n=302)\end{array}$ & $\begin{array}{c}\text { 30-42 years } \\
(n=170)\end{array}$ & $P$ value $^{\star}$ \\
\hline \multicolumn{5}{|l|}{ Clinical } \\
\hline Age (years) & $28.5 \pm 4.4$ & $25.8 \pm 2.8$ & $33.2 \pm 2.5$ & \\
\hline Amenorrhoea $(n) \dagger$ & $115(24 \%)$ & $72(24 \%)$ & $42(25 \%)$ & NS \\
\hline Cycle duration in oligomenorrhoeic patients (days) & $92 \pm 67$ & $93 \pm 68$ & $89 \pm 67$ & NS \\
\hline BMI $\left(\mathrm{kg} / \mathrm{m}^{2}\right)$ & $27.0 \pm 6.6$ & $27.2 \pm 7.0$ & $26.8 \pm 6.0$ & NS \\
\hline WHR & $0.8 \pm 0.01$ & $0.8 \pm 0.01$ & $0.8 \pm 0.01$ & NS \\
\hline \multicolumn{5}{|l|}{ Endocrine } \\
\hline LH (IU/I) & $7.6 \pm 4.6$ & $8.0 \pm 4.8$ & $6.9 \pm 4.3$ & 0.016 \\
\hline FSH (IU/l) & $4.8 \pm 1.5$ & $4.8 \pm 1.5$ & $4.8 \pm 1.4$ & NS \\
\hline $\mathrm{E}_{2}(\mathrm{pmol} / \mathrm{l})$ & $210 \pm 75$ & $212 \pm 73$ & $205 \pm 77$ & NS \\
\hline Inhibin B (ng/l) & $143 \pm 98$ & $150 \pm 101$ & $130 \pm 90$ & 0.05 \\
\hline Testosterone $(\mathrm{nmol} / \mathrm{l})$ & $2.4 \pm 1.0$ & $2.5 \pm 1.0$ & $2.2 \pm 0.9$ & 0.003 \\
\hline AD (nmol/l) & $14.3 \pm 6.9$ & $15.0 \pm 7.3$ & $13.0 \pm 6.0$ & 0.002 \\
\hline DHEAS (umol/l) & $7.1 \pm 3.4$ & $7.5 \pm 3.4$ & $6.5 \pm 3.2$ & 0.001 \\
\hline FAl & $6.9 \pm 7.1$ & $7.2 \pm 5.9$ & $6.3 \pm 8.6$ & NS \\
\hline SHBG (nmol/l) & $51.3 \pm 33.3$ & $49.5 \pm 31.9$ & $55.5 \pm 35.4$ & 0.06 \\
\hline Glucose $(\mathrm{mmol} / \mathrm{l})$ & $4.4 \pm 1.6$ & $4.3 \pm 1.6$ & $4.5 \pm 1.6$ & NS \\
\hline Insulin (mIU/l) & $15.1 \pm 12.0$ & $15.8 \pm 13.1$ & $13.9 \pm 9.8$ & NS \\
\hline Glucose/insulin (mmol/mlU) & $0.4 \pm 0.3$ & $0.4 \pm 0.3$ & $0.5 \pm 0.3$ & NS \\
\hline Cortisol (nmol/l) & $397 \pm 158$ & $400 \pm 159$ & $391 \pm 157$ & NS \\
\hline \multicolumn{5}{|l|}{ Transvaginal sonography } \\
\hline Mean ovarian volume (ml) & $9.3 \pm 4.6$ & $9.4 \pm 4.6$ & $9.1 \pm 4.6$ & NS \\
\hline Mean follicle number $(n)$ & $12.5 \pm 6.2$ & $12.9 \pm 6.0$ & $11.7 \pm 6.4$ & 0.05 \\
\hline $\operatorname{PCO}(n) \dagger$ & $99(21 \%)$ & $78(26 \%)$ & $22(13 \%)$ & 0.001 \\
\hline
\end{tabular}

*Student's $t$-test for normally distributed values; Mann-Witney $U$ test when not normally distributed. NS $=$ not significant.

†Percentages of women presenting with amenorrhoea or PCO were compared using Chi-square test.

Table 2 Bivariate correlations between age and clinical, endocrine and sonographic characteristics of 472 normogonadotrophic oligo-amenorrhoeic infertile patients of reproductive age.

\begin{tabular}{lcc}
\hline & $\boldsymbol{r}$ value $^{\star}$ & $\boldsymbol{P}$ value \\
\hline Clinical & & \\
Cycle duration† & -0.112 & 0.017 \\
$\mathrm{BMI}\left(\mathrm{kg} / \mathrm{m}^{2}\right)$ & -0.004 & $\mathrm{NS}$ \\
WHR & 0.114 & $\mathrm{NS}$ \\
& & \\
Endocrine & & \\
LH (IU/l) & -0.154 & 0.001 \\
FSH (IU/l) & 0.023 & $\mathrm{NS}$ \\
OE ${ }_{2}(\mathrm{pmol} / \mathrm{l})$ & -0.033 & $\mathrm{NS}$ \\
Inhibin B (ng/l) & -0.118 & 0.026 \\
Testosterone (nmol/l) & -0.194 & 0.001 \\
AD (nmol/l) & -0.170 & 0.001 \\
DHEAS ( $\mu$ mol/l) & -0.157 & 0.001 \\
FAl & -0.158 & 0.001 \\
SHBG (nmol/l) & -0.107 & 0.02 \\
Glucose (mmol/l) & 0.039 & $\mathrm{NS}$ \\
Insulin (mlU/l) & -0.126 & 0.015 \\
Glucose/insulin ratio (mmol/mlU) & 0.138 & 0.009 \\
Cortisol (nmol/l) & -0.009 & $\mathrm{NS}$ \\
& & \\
Transvaginal sonography & & \\
Mean ovarian volume (ml) & -0.026 & $\mathrm{NS}$ \\
Mean follicle number $(n)$ & -0.100 & 0.034 \\
Total stroma score* & -0.005 & $\mathrm{NS}$ \\
\hline
\end{tabular}

*Spearman's rank correlation.

†Defined as number of days between spontaneous menses (oligomenorrhoeic women only). NS=not significant. and biologically pertinent parameters such as cycle duration, LH, FSH, androgens and inhibin B are depicted in Figs 1-3.

No significant correlation between age and $\mathrm{E}_{2}$ levels was found; neither between age and BMI, WHR, mean ovarian volume, nor total stroma score (Table 2). No changes in correlations of all the aforementioned parameters with age were observed when patients were grouped and analysed according to the cycle duration (oligomenorrhoeic vs amenorrhoeic women; data not shown).

Covariance analysis to evaluate the relative contribution of BMI and WHR on the effect of age on the studied parameters revealed no differences in the strength of correlation among these variables (data not shown).

\section{Discussion}

Although abnormalities observed in PCOS have been studied extensively, little information is available concerning possible changes in clinical, endocrine and sonographic features associated with increasing age. In the current cross-sectional study in a large cohort of normogonadotrophic anovulatory infertile women, weak but statistically significant inverse correlations were observed between age and cycle duration, LH, androgens, SHBG, insulin, inhibin B and 


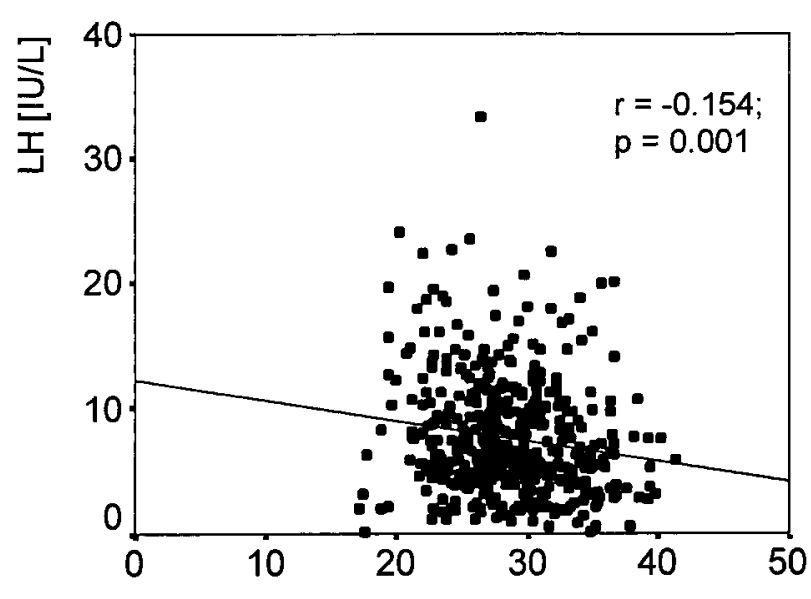

Age [yrs]

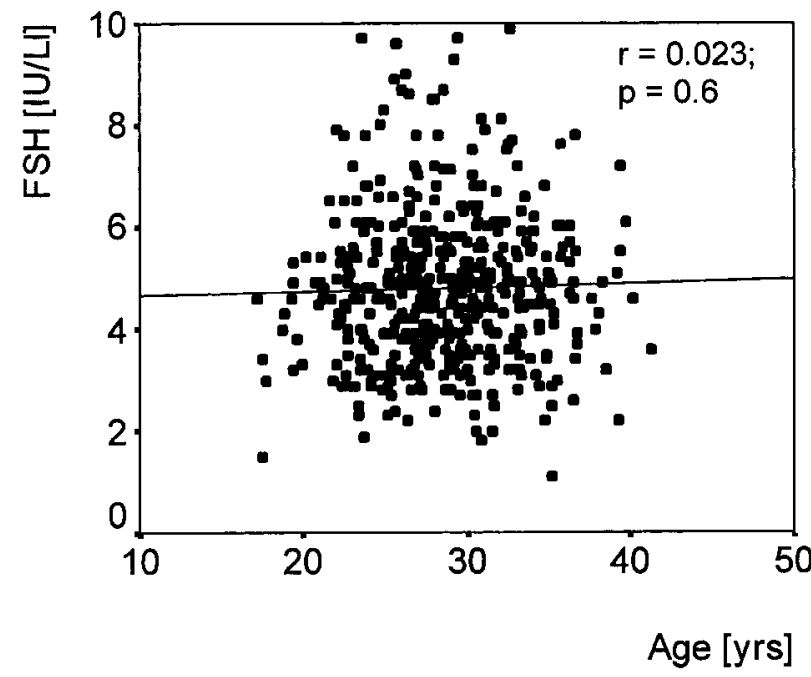

Figure 1 Bivariate correlations between $\mathrm{LH}$ and FSH serum concentrations with age in 472 normogonadotrophic oligo-amenorrhoeic infertile patients.

mean number of ovarian follicles. In addition, LH, testosterone, $\mathrm{AD}$, and DHEAS concentrations were decreased in women over 30 years compared with younger women. Moreover, the mean number of follicles was significantly decreased and polycystic ovaries were less frequently encountered in older women.

The slight decline in cycle duration with increasing age that we found may be related to the simultaneous fall in androgens. It is known that both normal weight and obese oligomenorrhoeic hirsute patients exhibit higher testosterone and FAI levels compared with normo-ovulatory hirsute women $(20,21)$. Thus, it seems that androgen levels are correlated more with menstrual pattern than with patient's weight. We could not confirm a positive correlation between age and BMI, as described previously (22). This may be explained by the inclusion of anovulatory rather than
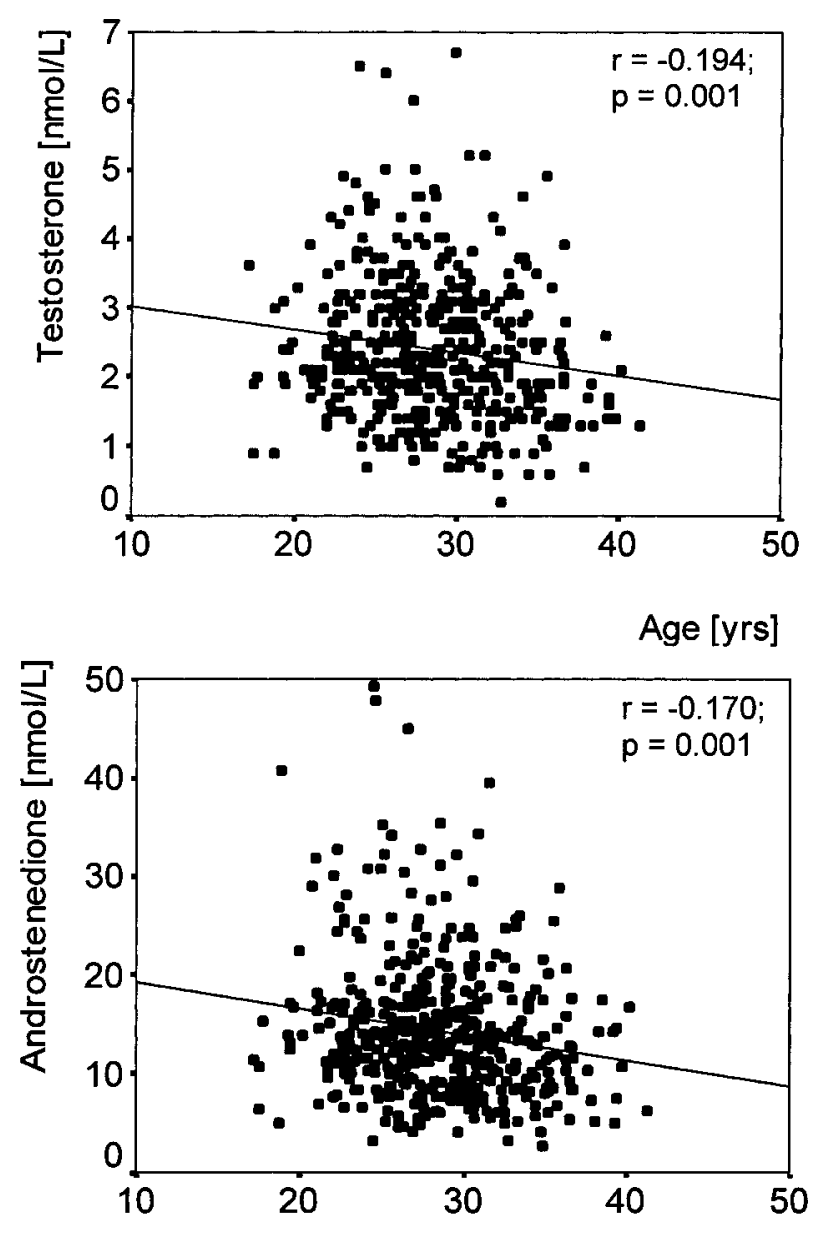

Age [yrs]

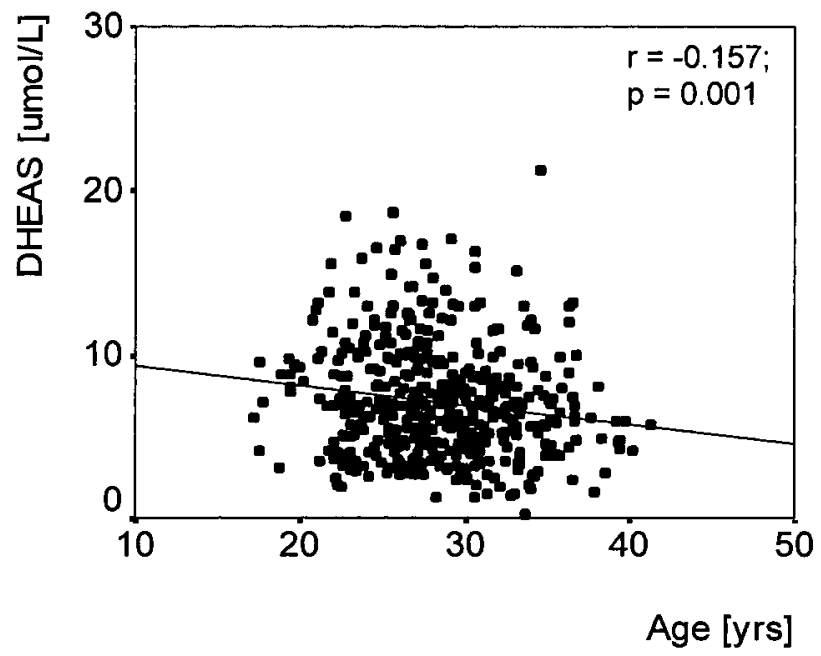

Figure 2 Bivariate correlations between testosterone, $A D$ and DHEAS serum concentrations with age in 472 normogonadotrophic oligo-amenorrhoeic infertile patients. 

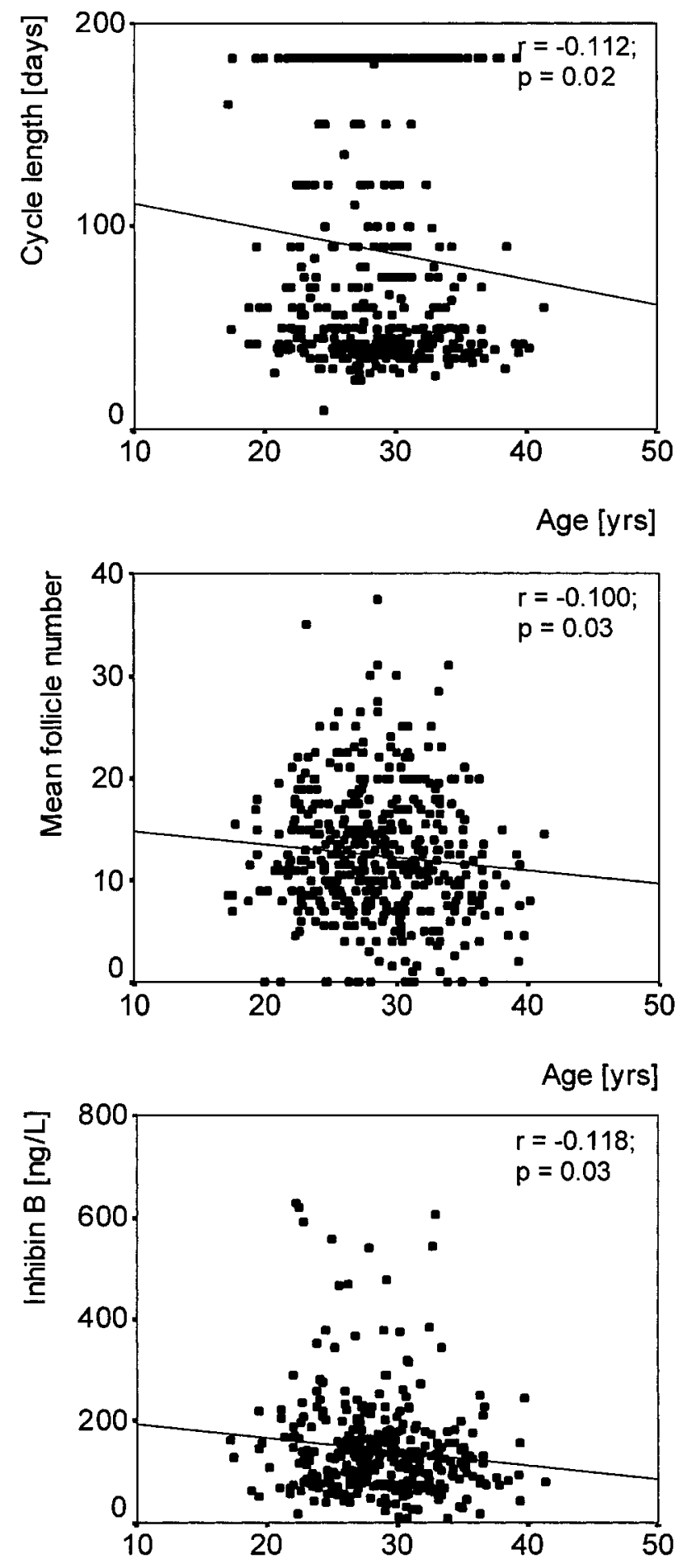

Age [yrs]

Figure 3 Bivariate correlations between parameters associated with normal ovarian ageing - cycle duration, mean follicle number and serum inhibin B concentrations - with age in 472 normogonadotrophic oligo-amenorrhoeic infertile patients. normally cycling women and by the relatively young age of the current study population.

Data from the present cross-sectional study do not provide strong evidence that the incidence of cycle abnormalities in PCOS women is related to their age. In contrast, other longitudinal studies $(11,12)$ suggested that cycle abnormalities tend to normalise with age. This discrepancy may again be due to differences in age of the patients studied, but also differences in study design, and criteria used to diagnose PCOS. Moreover, women with cycle abnormalities at advanced age are more likely to use steroid contraceptives and therefore their spontaneous cycle cannot be assessed in longitudinal follow-up studies. Indeed, the number of dropouts from reported follow-up studies is very high. However, it should be emphasised that the current cross-sectional study does not rule out cycle changes over time.

In normal women of reproductive age, the negative relationship of age with serum androgens has been established by previous investigators $(6,23)$. In addition, it has been shown that in hirsute hyperandrogenic women, the negative association between testosterone, AD, DHEAS levels and age is preserved (15). Our observations confirm these previous findings and suggest that the age of the patients should be taken into consideration when evaluating the hormonal profile of normogonadotrophic anovulatory infertile patients. The weak correlations found do not permit us to interpret these data as disease amelioration, but rather a physiological decline also observed in healthy women. It has been suggested that both adrenal and ovarian contributions to serum androgens decline with age (24).

In the current study, fasting serum insulin concentrations were not consistently increased and average levels were within the normal range as observed in other studies $(25,26)$. Hyperinsulinaemia is most prominent in obese PCOS patients (27) and the present study included both lean and obese women. A weak positive correlation between age and fasting glucose to insulin ratio was observed, suggesting that a slight amelioration of insulin resistance may occur with age. This is in accordance with a gradual decline in androgen levels with age since insulin may be directly involved in androgen production at the ovarian level (28).

LH concentrations were reported to remain unchanged in women with regular menstrual cycles, even above 45 years of age (29). Other studies reported a rise in $\mathrm{LH}$, almost simultaneous with FSH (30). The finding of a weak inverse relationship between age and $\mathrm{LH}$ in the current study in anovulatory women is unexpected and surprising. A statistically significant decrease in LH levels was also observed when young women were compared with those above 30 years of age. In a recent 10-year follow-up study (31) gonadotrophin levels were significantly reduced in PCOS 
patients using oestrogen-progestogen compounds, but showed no decline in non-users. Another study (13) was unable to find differences in gonadotrophin levels comparing adolescent and adult PCOS patients. Recent work from our group suggest that initial LH concentrations do not predict ovarian responsiveness to ovulation induction, indirectly opposing the concept that elevated LH levels are involved in ovarian dysfunction in these patients (18). Moreover, a single assessment of LH levels is of limited significance due to the timing of blood sampling, immunoassays used and the pulsatile nature of LH secretion (32). For all the aforementioned reasons, the pathophysiological relevance of the observed slight decline in LH with increasing age remains uncertain.

It is well established that in normal women the depletion of ovarian follicles continues during the reproductive years until the supply of resting primordial follicles is exhausted (1). Indeed, in normo-ovulatory women the number of antral follicles assessed by ultrasound decreases with age $(8,33)$. In PCOS only advanced stages of follicle maturation are arrested, resulting in an increased number of small antral follicles (34). No information is available concerning the extent of follicle depletion or menopausal age in PCOS women. In the current study in anovulatory women we found for the first time a reduction in follicle number with increasing age, in line with normoovulatory women. Combining these observations concerning LH, androgens and mean follicle numbers and defining PCOS as polycystic ovaries and elevated LH and androgen levels, the incidence of PCOS in normoovulatory infertile women may decrease over time.

The pattern of FSH and inhibin B secretion in normal women of reproductive age has previously been characterised $(1,4,30,35)$. FSH begins to rise at the age of 35 with a marked increase beyond 40 years. Inhibin B demonstrates a slight drop at the age of 35 and declines thereafter $(29,35)$. Moreover, inhibin B serum levels are normal in normogonadotrophic anovulatory women as well as in PCOS patients (36). In the current study, anovulatory women with elevated or reduced FSH were excluded since these groups represent different aetiological entities such as premature menopause. However, the normal range for FSH is wide (1-10 IU/l) and age-related changes may be established. From our data (showing no correlation of age with FSH and a weak inverse correlation with inhibin B) we can conclude that inhibin B secretion in normogonadotrophic anovulatory women follows the same pattern as in healthy women. A direct inverse correlation between inhibin B and FSH could not be confirmed.

In conclusion, the combined data of the current cross-sectional study do indicate appreciable changes in clinical, endocrine and ovarian characteristics associated with PCOS with increasing age in WHO class II anovulatory patients of reproductive age. It seems that observed minor changes follow the same pattern as in normo-ovulatory women of similar age. These changes may affect the observed incidence of PCOS. A longitudinal follow-up study should confirm these observations and should further establish the precise timing and magnitude of observed alterations.

\section{Acknowledgements}

The study was supported financially by the 'Stichting Voortplantingsgeneeskunde', Rotterdam, The Netherlands

\section{References}

1 Ahmed Ebbiary N, Lenton E \& Cooke I. Hypothalamic-pituitary ageing: progressive increase in $\mathrm{FSH}$ and $\mathrm{LH}$ concentrations throughout the reproductive life in regularly menstruating women. Clinical Endocrinology 199441 199-206.

2 te Velde E, Scheffer G, Dorland M, Broekmans F \& Fauser B. Developmental and endocrine aspects of normal ovarian aging. Molecular and Cellular Endocrinology 1998145 67-73.

3 Lenton E, Landgren B, Sexton L \& Harper R. Normal variation in the length of the follicular phase of the menstrual cycle: effect of chronological age. British Journal of Obstetrics and Gynaecology $198491681-684$.

4 Sherman B, West J \& Korenman S. The menopausal transition: analysis of LH, FSH, estradiol, and progesterone concentrations during menstrual cycles of older women. Journal of Clinical Endocrinology and Metabolism 197642 629-636.

5 Scott R \& Hofmann G. Prognostic assessment of ovarian reserve. Fertility and Sterility $1995631-11$.

6 Klein N, Illingworth P, Groome N, McNeilly A, Battaglia D \& Soules M. Decreased inhibin B secretion is associated with the monotropic FSH rise in older, ovulatory women: a study of serum and follicular fluid levels of dimeric inhibin $A$ and $B$ in spontaneous menstrual cycles. Journal of Clinical Endocrinology and Metabolism $1996 \mathbf{8 1} 2742-2745$.

7 Labrie F, Belanger A, Cusan L, Gomez J \& Candas B. Marked decline in serum concentrations of adrenal C19 sex steroid precursors and conjugated androgen metabolites during aging. Journal of Clinical Endocrinology and Metabolism 199782 23962402.

8 Scheffer G, Broekmans F, Dorland M, Habbema J, Looman C \& te Velde E. Antral follicle counts by transvaginal ultrasonography are related to age in women with proven natural fertility. Fertility and Sterility 199972 845-851.

9 van Santbrink E, Hop W \& Fauser B. Classification of normogonadotropic infertility: polycystic ovaries diagnosed by ultrasound versus endocrine characteristics of polycystic ovary syndrome. Fertility and Sterility $199767452-458$.

10 Dunaif A. Insulin action in the polycystic ovary syndrome. Endocrinology and Metabolism Clinics of North America 199928 341-359.

11 Dahlgren E, Johansson S, Lindstedt G, Knutsson F, Oden A, Janson PO, Mattson LA, Crona N \& Lundberg PA. Women with polycystic ovary syndrome wedge resected in 1956 to 1965: a long-term follow-up focusing on natural history and circulating hormones. Fertility and Sterility 1992 57 505-513.

12 Elting M, Korsen T, Rekers-Mombarg L \& Schoemaker J. Women with polycystic ovary syndrome gain regular menstrual cycles when ageing. Human Reproduction 200015 24-28.

13 Gulekli B, Turhan N, Senoz S, Kukner S, Oral H \& Gokmen O. Endocrinological, ultrasonographic and clinical findings in adolescent and adult polycystic ovary patients: a comparative study. Gynecologic Endocrinology 1993 7 273-277. 
14 Ruutiainen K, Erkkola R, Gronroos M \& Kaihola H. Androgen parameters in hirsute women: correlations with body mass index and age. Fertility and Sterility 198850 255-259.

15 Moran C, Knochenhauer E, Boots L \& Azziz R. Adrenal androgen excess in hyperandrogenism: relation to age and body mass. Fertility and Sterility 199971 671-674.

16 van Santbrink E, Hop W, van Dessel T, de Jong F \& Fauser B. Decremental follicle-stimulating hormone and dominant follicle development during the normal menstrual cycle. Fertility and Sterility $19956 \mathbf{6 4} 3-43$.

17 Pache T, Wladimiroff J, Hop W \& Fauser B. How to discriminate between normal and polycystic ovaries: transvaginal US study. Radiology $1992 \mathbf{1 8 3} 421-423$.

18 Imani B, Eijkemans $M$, te Velde E, Habbema J \& Fauser B. Predictors of patients remaining anovulatory during clomiphene citrate induction of ovulation in normogonadotropic oligoamenorrheic infertility. Journal of Clinical Endocrinology and Metabolism $1998 \mathbf{8 3} 2361-2365$.

19 Schipper I, de Jong F \& Fauser B. Lack of correlation between maximum early follicular phase serum follicle stimulating hormone concentrations and menstrual cycle characteristics in women under the age of 35 years. Human Reproduction 199813 1442-1448.

20 Zhang Y, Stern B \& Rebar R. Endocrine comparison of obese menstruating and amenorrheic women. Journal of Clinical Endocrinology and Metabolism 198458 1077-1083.

21 Cumming D \& Wall S. Non-sex hormone-binding globulin-bound testosterone as a marker for hyperandrogenism. Journal of Clinical Endocrinology and Metabolism $198561873-876$.

22 Evans W. Exercise and nutritional needs of elderly people: effects on muscle and bone. Gerodontology 199815 15-24.

23 Purifoy F, Koopmans L \& Tatum R. Steroid hormones and aging: free testosterone, testosterone and androstenedione in normal females aged 20-87 years. Human Biology 198052 181-191.

24 Maroulis G \& Abraham G. Ovarian and adrenal contributions to peripheral steroid levels in postmenopausal women. Obstetrics and Gynecology $1976 \mathbf{4 8} 150-154$.

25 Legro R, Finegood D \& Dunaif A. A fasting glucose to insulin ratio is a useful measure of insulin sensitivity in women with polycystic ovary syndrome [see comments]. Journal of Clinical Endocrinology and Metabolism $1998 \mathbf{8 3} 2694-2698$.

26 van Dessel T, Lee P, Faessen G, Fauser B \& Giudice L. Elevated serum levels of free insulin-like growth factor I in polycystic ovary syndrome. Journal of Clinical Endocrinology and Metabolism 199984 3030-3035.
27 Pasquali R, Casimirri F, Venturoli S, Antonio M, Morselli L, Reho S, Pezzoli A \& Paradisi R. Body fat distribution has weightindependent effects on clinical, hormonal, and metabolic features of women with polycystic ovary syndrome. Metabolism $1994 \mathbf{4 3}$ 706-713.

28 Dunaif A. Insulin resistance and the polycystic ovary syndrome: mechanism and implications for pathogenesis. Endocrine Reviews 199718 774-800.

29 MacNaughton J, Banah M, McCloud P, Hee J \& Burger H. Age related changes in follicle stimulating hormone, luteinizing hormone, oestradiol and immunoreactive inhibin in women of reproductive age. Clinical Endocrinology 199236 339-345.

30 Fitzgerald C, Seif M, Killick S \& Elstein M. Age related changes in the female reproductive cycle. British Journal of Obstetrics and Gynaecology 1994101 229-233.

31 Pasquali R, Gambineri A, Anconetani B, Vicennati V, Colitta D, Caramelli E, Casimirri F \& Morselli-Labate AM. The natural history of the metabolic syndrome in young women with the polycystic ovary syndrome and the effect of long-term oestrogenprogestagen treatment. Clinical Endocrinology 199950 517-527.

32 Fauser B \& de Jong F. Gonadotropins in polycystic ovarian syndrome. Annals of the New York Academy of Science 1993687 150-161.

33 Ruess M, Kline J, Santos R, Levin B \& Timor-Tritsch I. Age and the ovarian follicle pool assessed with transvaginal ultrasonography. American Journal of Obstetrics and Gynecology $1996 \mathbf{1 7 4}$ 624-627.

34 Fauser B. Observations in favor of normal early follicle development and disturbed dominant follicle selection in polycystic ovary syndrome. Gynecologic Endocrinology 19948 75-82.

35 Danforth DR, Arbogast LK, Mroueh J, Kim MH, Kennard EA, Seifer DB \& Friedman CI. Dimeric inhibin: a direct marker of ovarian aging. Fertility and Sterility 1998 70 119-123.

36 Laven J, Imani B, Eijkemans M, de Jong F \& Fauser B. Absent biologically relevant associations between serum inhibin $\mathrm{B}$ concentrations and characteristics of polycystic ovary syndrome in normogonadotrophic anovulatory infertility. Human Reproduction $2001161359-1364$.

Received 2 May 2001

Accepted 8 August 2001 